\title{
CHAVEAMENTO CONTROLADO DE LINHAS DE TRANSMISSÃO: UMA ABORDAGEM EM TEMPO REAL VIA RTDS ${ }^{\text {TM }}$
}

\author{
Karcius Marcelus Colaço Dantas* \\ karcius@dee.ufcg.edu.br \\ Damásio Fernandes Jr. ${ }^{*}$ \\ damasio@dee.ufcg.edu.br
}

\author{
Luiz Carlos de Alcântara Fonseca ${ }^{\dagger}$ \\ lfonseca@chesf.gov.br \\ *Universidade Federal de Campina Grande - CEEI/UAEE/GSE \\ Av. Aprígio Veloso, 882, Bodocongó, CEP 58.429-140, Campina Grande, Paraíba, Brasil \\ ${ }^{\dagger}$ Companhia Hidro Elétrica do São Francisco \\ R. Quinze de Março, 50, Bongi, CEP 58.761-901, Recife, Pernambuco, Brasil
}

\author{
Washington Luiz Araújo Neves* \\ waneves@dee.ufcg.edu.br \\ Gustavo de Alcântara Cardoso* \\ gustavoacardoso@gmail.com
}

\begin{abstract}
Transmission Line Controlled Switching: A Real Time Approach via RTDS

The goal of this work is to present a method for controlled switching of transmission line circuit-breakers and to develop a device to control the closing of its contacts. The switching strategy consists on finding a suitable circuit breaker making instant and it is based on a very simple zero crossing algorithm. For the first time, the method is evaluated in real time using a RTDS ${ }^{\mathrm{TM}}$ (Real Time Digital Simulator) and its implementation is accomplished in two ways: first, it is used the CBuilder tool of the RTDS ${ }^{\mathrm{TM}}$ and second, a DSP (Digital Signal Processor) is used as a control device. Data from the CHESF power system are used to produce case studies. Different line switching events are analyzed: closing and re-closing of shunt compensated lines taking into account trapped charge effects. The performance of the proposed method is compared to the pre-insertion resistors method. The simulations attest the efficiency of the controlled switching, which is economically more attractive.
\end{abstract}

KEYWORDS: Controlled Switching, DSP, RTDS ${ }^{\mathrm{TM}}$, Switching Overvoltages, Transmission Lines.

Artigo submetido em 17/10/2009 (Id.: 01064)

Revisado em 30/01/2010, 11/06/2010

Aceito sob recomendação do Editor Associado Prof. Eduardo N. Asada

\section{RESUMO}

O objetivo deste trabalho é apresentar um método para chaveamento controlado de disjuntores de linhas de transmissão e desenvolver um dispositivo para controlar o fechamento dos seus contatos. A estratégia de chaveamento consiste em encontrar um instante apropriado para o fechamento do disjuntor e é baseada em um algoritmo simples de detecção de passagem por zero. Pela primeira vez, o método é avaliado em tempo real usando um RTDS ${ }^{\mathrm{TM}}$ (Real Time Digital Simulator) e sua implementação se dá de duas formas: na primeira, utiliza-se a ferramenta $C B u i l d e r$ do $\operatorname{RTDS}^{\mathrm{TM}}$ e na segunda, utiliza-se um DSP (Digital Signal Processor) como dispositivo de controle. Dados do sistema elétrico da CHESF são utilizados para estudos de caso. Diferentes operações de chaveamento são analisadas: energização e religamento de linhas com compensação em derivação, considerando os efeitos da carga residual. O desempenho do método proposto é comparado com o método de resistores de pré-inserção. As simulações confirmam a eficiência do chaveamento controlado, o qual é economicamente mais atrativo.

PALAVRAS-CHAVE: Chaveamento Controlado, DSP, Linhas de Transmissão, RTDS ${ }^{\mathrm{TM}}$, Sobretensões de Manobra. 


\section{INTRODUÇÃO}

Sobretensões de manobra influenciam diretamente a coordenação de isolamento de sistemas elétricos em extra-alta tensão caracterizados por longas linhas de transmissão (LTs), a exemplo do Sistema Interligado Nacional (SIN). Desta forma, estudos relacionados aos meios para reduzir estas sobretensões são de extrema importância para o setor elétrico. Tradicionalmente, disjuntores equipados com resistores de pré-inserção (RPIs) têm sido utilizados para este fim (Wagner and Bankoske, 1967). Porém, com o avanço da tecnologia e o maior emprego da eletrônica nos sistemas de potência, técnicas de chaveamento controlado destacam-se como alternativa (CIGRE Task Force 13.00.1, 1995; CIGRE Task Force 13.00.1, 1996). Chaveamento controlado é o termo utilizado para descrever o uso de equipamentos eletrônicos para controlar o fechamento e abertura mecânica dos contatos dos disjuntores (CIGRE Working Group 13.07, 1999a). Devido à viabilidade econômica e aos benefícios auferidos, esse tema tem despertado interesse tanto das companhias quanto dos fabricantes de disjuntores (ABB, 2009; Tsutada et al., 2002).

A utilização do chaveamento controlado em LTs elimina a necessidade do uso de RPIs, simplificando significativamente o projeto dos disjuntores e barateando seus custos de fabricação e manutenção (CIGRE Working Group 13.07, 1999b). Nos últimos anos, diversos trabalhos atestaram a eficiência deste método (Froehlich et al., 1997a; Ito, 2002; Mestas and Tavares, 2007; Dantas et al., 2009). No entanto, a maioria dos trabalhos não apresentam metodologias para sua implementação além de que poucos tiveram um caráter experimental objetivando a construção de um dispositivo de controle do chaveamento (Cardoso, 2009). Neste trabalho, um método para chaveamento controlado de linhas de transmissão é proposto tendo como objetivo o desenvolvimento de um dispositivo capaz de controlar o fechamento dos contatos dos disjuntores. A estratégia de chaveamento apresentada consiste em determinar instantes apropriados para este fechamento e é baseada em um algoritmo simples de detecção de passagem por zero. Para tanto, a avaliação do método em tempo real se torna imprescindível.

A análise do comportamento de sistemas elétricos e de dispositivos de controle em tempo real era feita majoritariamente por meios analógicos através de modelos de sistemas elétricos em miniatura, conhecidos como Analisadores de Rede ou Transient Network Analysers (TNA) (Doi et al., 1990). Com o avanço da tecnologia computacional e o advento de dispositivos cada vez mais eficazes para o processamento digital, a implementação de algoritmos para solução digital de transitórios eletromagnéticos em tempo real tornou-se viável e equipamentos para simulação digital em tempo real foram desenvolvidos, a exemplo do RTDS ${ }^{\mathrm{TM}}$ (Real-Time Digital Simulator) (Kuffel et al., 1995).
Recentemente, a Universidade Federal de Campina Grande adquiriu um RTDS ${ }^{\mathrm{TM}}$ (figura 1), o qual se configura como uma combinação de hardware e software funcionando como um TNA digital. O RTDS ${ }^{\mathrm{TM}}$ utiliza os mesmos algoritmos e equações encontradas em programas do tipo EMTP (Electromagnetic Transients Program) (Dommel, 1996). Porém, a simulação é considerada em tempo real devido às soluções do sistema elétrico serem obtidas em um intervalo de tempo muito menor que as constantes de tempo do sistema. O RTDS ${ }^{\mathrm{TM}}$ apresenta uma interface para entrada e saída de dados do sistema elétrico, tais como trips de relés e sinais de tensão e corrente, permitindo assim a comunicação com equipamentos externos (Forsyth et al., 2004).

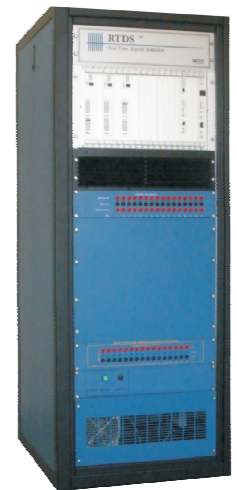

Figura 1: Rack do RTDS ${ }^{\text {TM }}$.

Neste trabalho, a implementação do método para chaveamento controlado de linhas de transmissão se dá de duas maneiras:

- Utilizando a ferramenta computacional CBuilder, a qual se configura como um ambiente de criação de novos componentes que podem ser incorporados às simulações e interagir com os componentes já fornecidos pelo RTDS $^{\mathrm{TM}}$ (Ouellette et al., 2008).

- Utilizando um DSP (Digital Signal Processor) para o desenvolvimento de um dispositivo de controle, externo ao RTDS ${ }^{\mathrm{TM}}$, capaz de sincronizar os instantes de fechamento dos pólos do disjuntor.

Dados do SIN referentes ao sistema elétrico da Companhia Hidro Elétrica do São Francisco (CHESF) são utilizados para estudos de caso e diversas condições de operação de chaveamento são analisadas: energização e religamento tripolar de linhas com compensação em derivação, considerando os efeitos da compensação reativa em paralelo e da carga residual nas linhas. Os resultados comprovaram a eficiência do chaveamento controlado, o qual apresentou resultados compatíveis com os obtidos com resistores de pré-inserção. 


\section{FUNDAMENTOS DO CHAVEAMENTO CONTROLADO}

Normalmente, o comando para energização ou religamento de LTs é realizado em um instante aleatório $\left(t_{\text {comando }}\right)$ em relação à forma de onda da tensão entre os contatos do disjuntor, a qual representa o sinal de referência para o chaveamento controlado. Ainda, a manobra somente é efetivada após o período denominado tempo de operação do disjuntor $\left(T_{\text {operacao }}\right)$, que representa o intervalo de tempo entre a energização do circuito de fechamento do disjuntor e o acoplamento físico entre os seus contatos. Um valor típico para este intervalo é $50 \mathrm{~ms}$ (Froehlich et al., 1997a).

Na figura 2 é ilustrada a estratégia para controle de manobras de fechamento. O procedimento consiste em controlar o instante $t_{\text {comando }}$ atrasando-o por um intervalo de tempo $T_{\text {atraso }}$ de forma que o instante apropriado para o fechamento dos pólos do disjuntor $\left(t_{\text {otimo }}\right)$ ocorra em um instante $T_{\text {atraso }}+T_{\text {operacao }}$ depois de $t_{\text {comando }}$. O intervalo $T_{\text {atraso }}$ adicionado ao instante $t_{\text {comando }}$ pode ser determinado por dois subintervalos:

- $T_{\text {calculo }}$ : intervalo de processamento computacional para determinação de $t_{\text {otimo; }}$;

- $T_{\text {sincronizacao }}$ : intervalo para sincronização com $t_{\text {otimo }}$ considerando $T_{\text {operacao }}$.

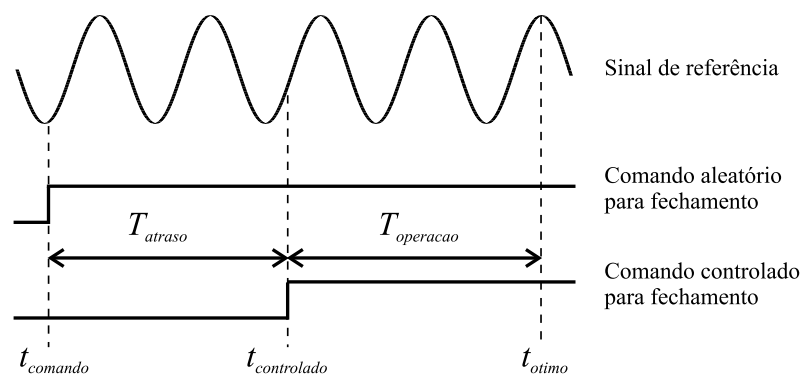

Figura 2: Temporização do chaveamento controlado.

\subsection{Energização de LTs}

Energizações de LTs são manobras programadas que podem ocorrer rotineiramente em sistemas de potência. Além disso, durante uma manobra de energização não há cargas residuais nas linhas de transmissão, sendo esta a condição mais simples para a realização do chaveamento controlado. Neste caso, $t_{\text {otimo }}$ ocorre na passagem por zero da tensão do lado da fonte. Assim, para cada fase, basta o monitoramento deste sinal de tensão o qual será o sinal de referência para o controle do chaveamento. Na figura 3, estão indicados através de setas os possíveis instantes apropriados para a energização de uma fase da LT.

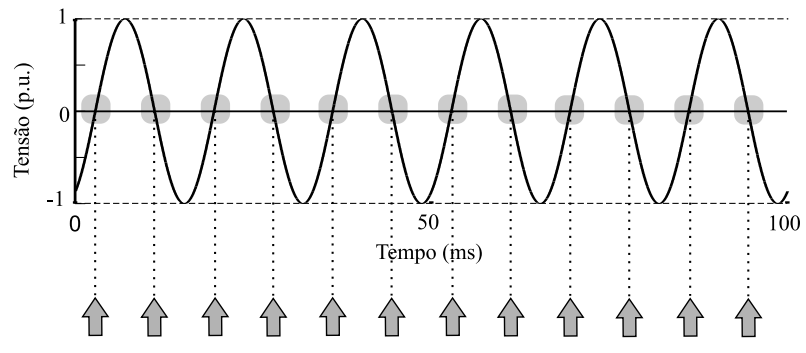

Figura 3: Tensão entre os contatos do disjuntor para LTs sem carga residual.

\subsection{Religamento de LTs com Compensa- ção em Derivação}

Operações de religamento de LTs são normalmente efetivadas com a presença de cargas residuais. Para linhas com compensação reativa em derivação, após a sua abertura, a carga residual apresenta uma oscilação devido ao circuito formado entre a capacitância da linha e a indutância dos reatores em derivação. Esta oscilação tem uma frequência menor que a frequência nominal do sistema, com valores entre 30 e $55 \mathrm{~Hz}$, a depender do grau de compensação da linha (Froehlich et al., 1997a).

Neste caso, o sinal de referência é a tensão entre os contatos do disjuntor, cuja forma de onda depende consequentemente do grau de compensação da linha de transmissão. Para graus de compensação elevados (figura 4), a tensão entre os contatos do disjuntor apresenta uma forma de onda com pulsação bem definida, enquanto que graus de compensação menores apresentam uma forma de onda mais complexa (figura 5). Em ambos os casos, os instantes apropriados para o chaveamento de cada fase, os quais também estão destacados nas figuras 4 e 5, ocorrem na passagem por zero do sinal de tensão entre os contatos do disjuntor e no período de menor pulsação deste sinal. Com isto, pode-se minimizar o efeito do pré-arco no momento do fechamento dos contatos do disjuntor, aumentando assim a confiabilidade do método (Froehlich et al., 1997b).

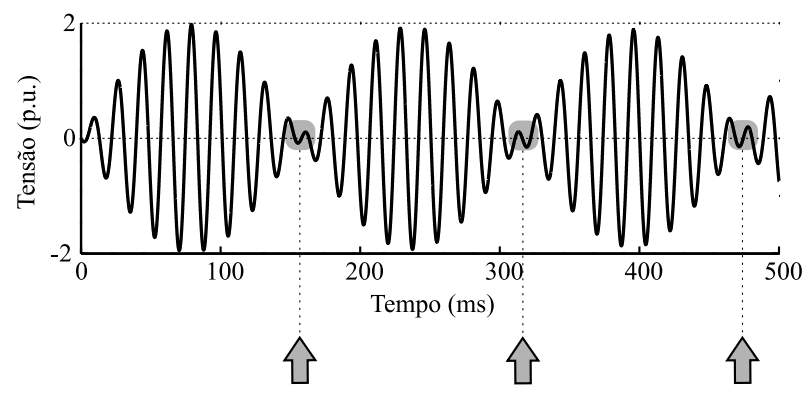

Figura 4: Tensão entre os contatos do disjuntor para LTs com grau de compensação de $80 \%$. 


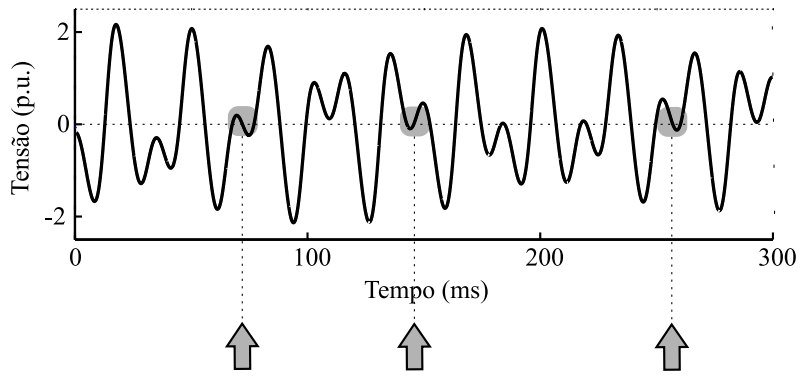

Figura 5: Tensão entre os contatos do disjuntor para LTs com grau de compensação de $30 \%$.

\section{MÉTODO PROPOSTO}

Métodos para chaveamento controlado de linhas com compensação reativa em derivação podem ser encontrados na literatura (Froehlich et al., 1997a). Porém, provavelmente devido a questões de propriedade intelectual, estes métodos não apresentam claramente os algoritmos para determinação dos instantes apropriados para o chaveamento dos pólos dos disjuntores.

Neste trabalho apresenta-se de forma detalhada um método para chaveamento controlado de LTs, o qual é baseado em um algoritmo simples de detecção de passagem por zero e consiste em estimar os sinais de referência em instantes futuros, possibilitando a determinação de instantes apropriados para o fechamento de cada fase da linha assim que o comando para a operação do disjuntor ocorra. Estes instantes são definidos como aqueles em que o sinal de tensão entre os contatos do disjuntor é zero e são determinados de forma que o intervalo entre o instante de fechamento da primeira fase e o instante de fechamento da última fase seja o menor possível.

Este forma de definição dos instantes apropriados para o chaveamento se configura como uma contribuição proposta no trabalho e acarreta na redução da distorção causada nos sinais de referência devido à tensão induzida no lado da linha pelo acoplamento eletromagnético entre as fases, o que aumenta a eficiência da técnica de chaveamento controlado (Dantas et al., 2008). Na figura 6, é apresentado o diagrama de blocos que descreve o funcionamento do método. Uma discussão sobre este diagrama é apresentada a seguir.

\subsection{Descrição do Método}

\subsubsection{Filtragem e Amostragem}

O Sistema de Controle do Chaveamento (SCC) recebe do sistema elétrico de potência os sinais de tensão relativos aos sinais de referência requeridos para a realização do chaveamento controlado. Em seguida, com o objetivo de minimizar o efeito de aliasing e atenuar componentes de alta frequência, um filtro digital Butterworth passa-baixa de terceira or-

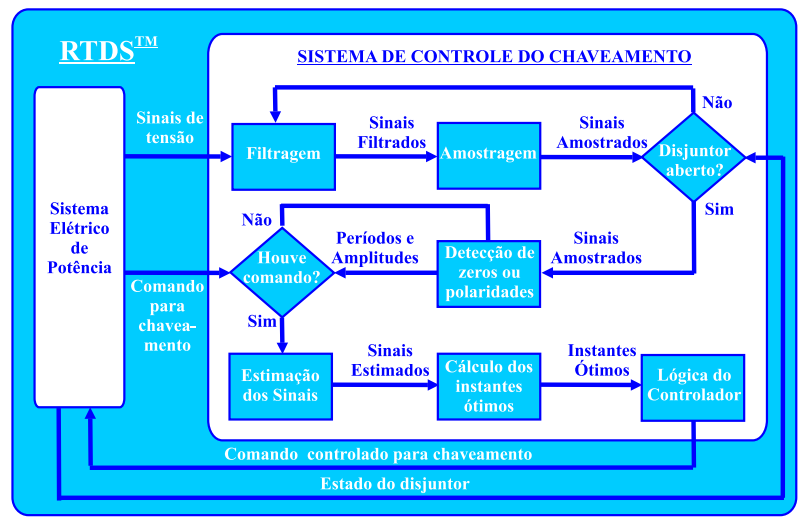

Figura 6: Método proposto: diagrama de blocos.

dem foi projetado e aplicado a estes sinais. Uma descrição sucinta deste tipo de filtro pode ser encontrada em livros de processamento de sinais como (Oppenheim and Schafer, 1989). A figura 7 é uma representação gráfica das especificações de projeto de um filtro Butterworth passa-baixa, em que $|A(j \omega)|_{d B}$ representa a atenuação do sinal (em dB) em função da frequência $\omega$. As freqüências $\omega_{p}$ e $\omega_{s}$ dividem as freqüências nas faixas de passagem, transição e corte. A frequiência $\omega_{c}$ é denominada de freqüência de corte do filtro e representa a freqüência na qual a atenuação do filtro é de $3 \mathrm{~dB}$. As variáveis $A_{\max }$ e $A_{\min }$ representam as atenuações nas faixas de passagem e corte, respectivamente.

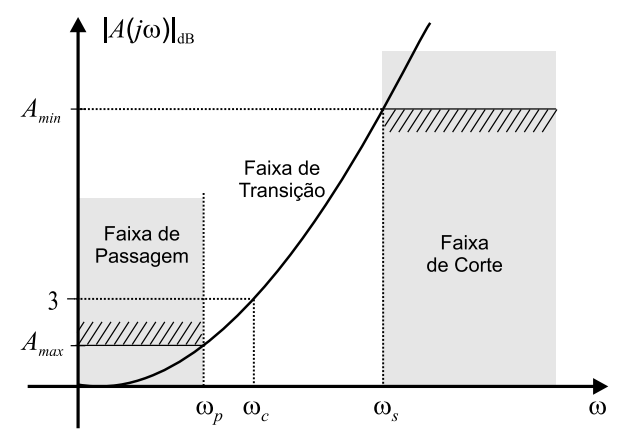

Figura 7: Especificações de projeto de um filtro Butterworth passa-baixa.

Como a faixa de frequência de interesse é de 30 a $60 \mathrm{~Hz}$, as seguintes especificações foram consideradas para o projeto do filtro: $f_{p}=150 \mathrm{~Hz} ; f_{s}=240 \mathrm{~Hz} ; A_{\max }=1$ $\mathrm{dB} ; A_{\text {min }}=60 \mathrm{~dB}$, em que $\omega_{p}=2 \pi f_{p}$ e $\omega_{s}=2 \pi f_{s}$. Com base nestes parâmetros, calcula-se a freqüência de corte $f_{c}=187,89 \mathrm{~Hz}$, a partir da equação 1 , em que $n$ é a ordem do filtro e $\omega_{c}=2 \pi f_{c}$ (Oppenheim and Schafer, 1989). 


$$
\omega_{c}=\frac{\omega_{p}}{\left(10^{0,1 A_{\max }}-1\right)^{\frac{1}{2 n}}}
$$

Após a filtragem, os sinais são amostrados a uma frequência de $960 \mathrm{~Hz}$, a qual é suficiente para que o sinal possa ser reproduzido integralmente sem erro de aliasing e é comumente utilizada em sistemas de proteção digital (Schweitzer and Hou, 1993).

\subsubsection{Detecção de Zeros ou Polaridades}

A partir do instante em que há a desenergização da linha de transmissão, deve-se realizar a detecção das passagens por zero dos sinais senoidais do lado da fonte e do lado da linha (para religamento de LTs com compensação em derivação). Uma passagem por zero é detectada sempre que um sinal senoidal muda de polaridade entre duas amostras consecutivas. Com isso, os períodos destes sinais são determinados através da detecção de duas passagens por zero consecutivas. Suas amplitudes são determinadas detectando-se o valor de pico entre dois zeros consecutivos.

\subsubsection{Estimação dos Sinais}

Ao receber o comando para chaveamento do disjuntor, o SCC estima os sinais de referência em instantes futuros baseandose nos últimos valores determinados para os períodos, amplitudes e passagens por zero dos sinais senoidais. Na figura 8 é ilustrado o procedimento para estimação dos sinais de referência em instantes futuros.

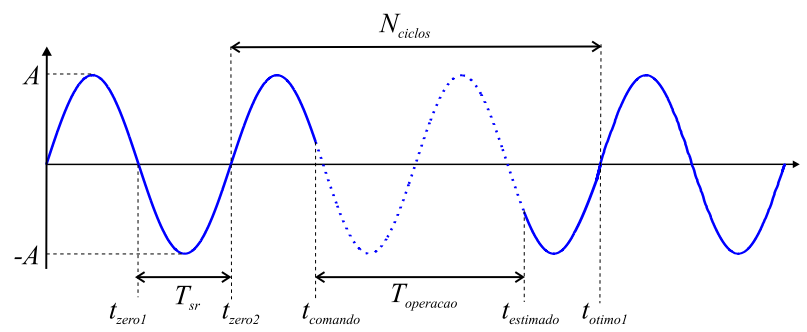

Figura 8: Estimação dos sinais de referência em instantes futuros.

Com o comando para o chaveamento do disjuntor ocorrendo no instante $t_{\text {comando }}, t_{\text {zero } 2}$ torna-se o último instante de passagem por zero do sinal de referência. Assim, considerando $A$ como a amplitude do sinal e $T_{s r}$ como meio período do mesmo, o sinal de referência $\left(s_{r e f}\right)$ é estimado após o período $T_{\text {operacao }}$, referente ao tempo de operação do disjuntor, da seguinte maneira:

$$
s_{r e f}(t)=A \cdot \operatorname{sen}\left(\omega_{s r} \cdot(t+\Delta T)\right) .
$$

Onde,

$$
\omega_{s r}=\frac{\pi}{T_{s r}},
$$

e

$$
\Delta T=T_{\text {operacao }}+\left(t_{\text {comando }}-t_{\text {zero } 2}\right) .
$$

Desta forma, o primeiro instante futuro estimado é ilustrado

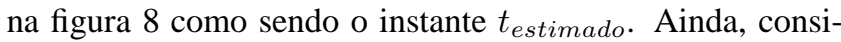
derando que o instante apropriado para a efetivação da manobra de chaveamento seja na passagem por zero do sinal, o primeiro instante disponível para o chaveamento ocorre em $t_{\text {otimo } 1}$.

\subsubsection{Cálculo dos Instantes Ótimos}

Uma vez que os sinais de referência são estimados em instantes futuros, pode-se determinar uma lista de possíveis instantes apropriados para o fechamento de cada fase de acordo com a condição de operação da linha de transmissão. Para a manobra de energização, como já exposto anteriormente, os instantes apropriados para chaveamento ocorrem na passagem por zero da tensão do lado da fonte e a forma de determinação destes instantes é descrita a seguir.

Com base na figura 8 e na equação 4 , tem-se que o número de ciclos (relativos a meio período $T_{s r}$ do sinal senoidal do lado da fonte) entre o último instante de passagem por zero do sinal e o primeiro instante apropriado a ser determinado é dado pela equação 5 , onde int sup $(x)=x$ quando $x$ for um número inteiro, ou caso contrário, é igual ao número inteiro imediatamente superior a $x$. Consequentemente, o primeiro instante apropriado para o chaveamento será dado pela equação 6 . E os próximos instantes se repetem a cada $T_{s r}$ segundos.

$$
N_{\text {ciclos }}=\operatorname{int}_{\text {sup }}\left(\frac{\Delta T}{2 \cdot T_{s r}}\right) \text {. }
$$

$$
t_{\text {otimo } 1}=t_{\text {zero } 2}+N_{\text {ciclos }} \cdot\left(2 T_{\text {sr }}\right) .
$$

Para a manobra de religamento de uma LT com compensação em derivação, devido a natureza oscilatória da carga residual, o procedimento para determinação dos instantes apropriados para o chaveamento torna-se um pouco mais complexo. Tal procedimento é descrito a seguir com auxílio da figura 9, a qual é meramente ilustrativa. Nesta figura, o sinal de tensão do lado da fonte possui a freqüência fundamental do sistema enquanto que o sinal do lado da linha possui uma frequência natural que depende do grau de compensação da linha. 


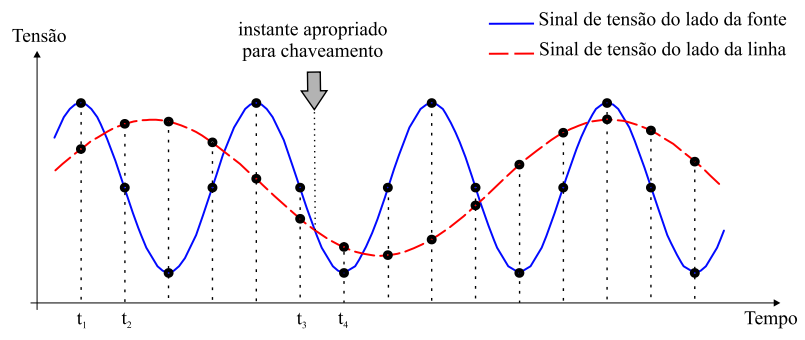

Figura 9: Estimação dos instantes ótimos para a manobra de religamento de linhas com compensação.

Com o intuito de reduzir os esforços requeridos para estimar os instantes apropriados para o religamento de LTs com compensação em derivação, as tensões do lado da fonte e do lado da linha são analisadas separadamente, sendo necessário o monitoramento dos dois sinais. Desta forma, apenas sinais senoidais são avaliados ao invés de sinais mais complexos como os das figuras 4 e 5 . Em seguida, estes sinais são postos em conjunto, obtendo assim o sinal de referência para esta condição de operação da linha.

Inicialmente, de posse dos dados relativos aos períodos, amplitudes e últimos instantes de passagem por zero dos sinais de tensão do lado da linha e do lado da fonte, os instantes futuros para cada sinal (ilustrados na figura 9 por círculos em negrito) são determinados a partir da equação 2 . Um passo de cálculo de 1/960 segundos é considerado tomando como base a frequência de amostragem de $960 \mathrm{~Hz}$ utilizada neste trabalho. Para representar os sinais senoidais do lado da linha e do lado da fonte entre cada dois instantes futuros estimados, é realizada uma interpolação linear. Em seguida os segmentos de reta são postos em conjunto, de forma a verificar se eles interceptam-se no intervalo definido por dois instantes futuros. Caso os segmentos se interceptem, determinase as suas derivadas (inclinações). Se os valores das derivadas tiverem o mesmo sinal (positivo ou negativo), o instante onde estes segmentos de reta se interceptam é definido como um instante apropriado para o chaveamento. Caso contrário, conclui-se que apesar de haver um instante onde o sinal de referência é zero, este instante não é considerado um instante apropriado pois não se situa no período de menor pulsação deste sinal.

Desta forma, pode-se observar na figura 9 que entre os instante $t_{3}$ e $t_{4}$ encontra-se um instante apropriado para o chaveamento, o qual é indicado por uma seta. Já entre os instantes $t_{1}$ e $t_{2}$, apesar de os sinais se interceptarem neste intervalo, o instante em que ocorre este cruzamento não é considerado como um instante apropriado.

\subsubsection{Lógica do Controlador}

Uma vez que uma lista de instantes apropriados para o religamento de cada fase é determinada e tomando como referência o comando para o chaveamento do disjuntor, a Lógica do Controlador atua atrasando este comando por um intervalo de tempo necessário para a realização do chaveamento de cada fase em um instante futuro apropriado. Assim, de acordo com a figura 2 e considerando que o tempo de operação do disjuntor apresenta uma dispersão estatística em relação ao tempo nominal de operação do mesmo ( $\left.T_{\text {nominal }}\right)$, a qual é difícil de ser estimada devido às incertezas usuais de fechamento hidráulico mecânico, o tempo de atraso é determinado por (7).

$$
T_{\text {atraso }}=\left(t_{\text {otimo }}-t_{\text {comando }}\right)-T_{\text {nominal }} .
$$

\subsection{Acoplamento Eletromagnético}

O acoplamento eletromagnético entre as fases das LTs pode ocasionar desvios significativos nos sinais de tensão utilizados para determinação dos instantes apropriados para o chaveamento, resultando em perda de eficiência na redução de sobretensões por meio do chaveamento controlado (CIGRE Working Group 13.07, 1999a). Este fenômeno ocorre, pois quando o primeiro pólo do disjuntor é fechado, uma tensão é induzida nas outras fases, e o mesmo acontece com a terceira fase após a segunda fase ser energizada.

A figura 10, obtida através de simulação digital de uma manobra de religamento de uma linha de transmissão com compensação reativa em derivação, ilustra os desvios nos sinais de tensão entre os contatos do disjuntor com relação aos instantes ótimos previstos para o chaveamento de cada fase. Pode-se observar que o efeito do acoplamento é mais significativo para a terceira fase do que para a segunda, resultando em um desvio maior em relação ao instante apropriado previsto para a terceira fase.

Com o intuito de reduzir a distorção, na tensão do lado da linha, causada pelo efeito do acoplamento eletromagnético entre as fases, os instantes apropriados para o chaveamento são determinados de forma que o intervalo entre o instante de fechamento do primeiro e do último pólo do disjuntor seja o menor possível.

\section{IMPLEMENTAÇÃO}

A implementação do método proposto para chaveamento controlado de linhas de transmissão se deu visando sua aplicação em tempo real. O RTDS ${ }^{\mathrm{TM}}$, descrito anteriormente, foi utilizado para este objetivo e o método foi implementado de duas formas distintas. Na primeira, a implementação se deu a 


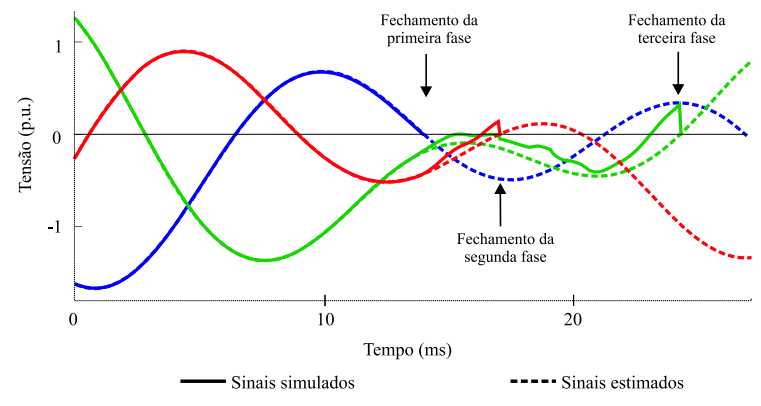

Figura 10: Efeito do acoplamento eletromagnético.

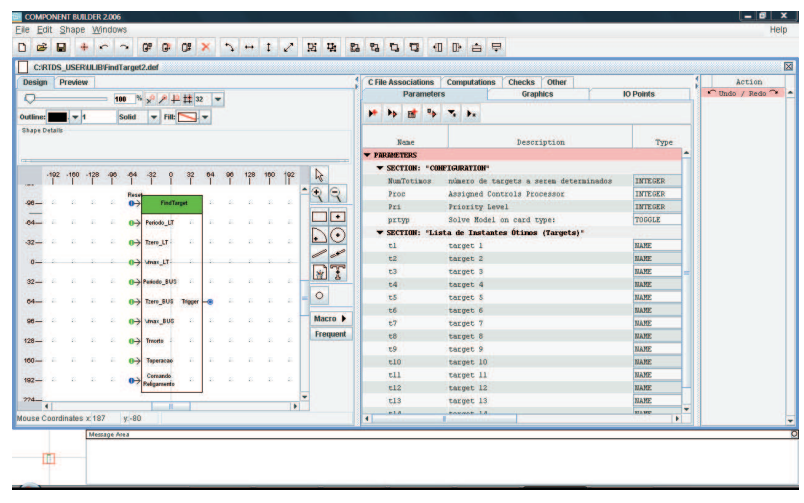

Figura 11: Módulo CBuilder.

nível de software, no próprio RTDS ${ }^{\mathrm{TM}}$, através da ferramenta CBuilder. Na segunda, o método foi implementado em hardware, mais especificamente em um DSP (Digital Signal Processor), caracterizando-se como um protótipo para um dispositivo de controle do chaveamento. A seguir, detalhes das ferramentas utilizadas na implementação são apresentados.

\subsection{Implementação em Software via CBuilder}

Diferentes níveis de software compõem o RTDS ${ }^{\mathrm{TM}}$. No nível mais baixo estão os modelos dos componentes de sistemas elétricos (linhas de transmissão, transformadores, geradores, etc), os quais são baseados nos algoritmos e equações encontradas em programas do tipo EMTP, porém otimizados para simulação em tempo real. Já no nível mais alto, encontrase a GUI (Graphical User Interface) denominada RSCAD. Esta interface permite a construção e elaboração de circuitos e componentes do sistema, bem como a simulação dos mesmos e a visualização dos resultados, os quais podem ser gravados e documentados (Forsyth et al., 2004).

Ainda, o RSCAD fornece o módulo Component Builder (CBuilder), mostrado na figura 11, o qual se constitui como um ambiente de criação de novos componentes de controle e do sistema elétrico mediante a implementação, por meio da linguagem de programação $\mathrm{C}$, de modelos e algoritmos desenvolvidos pelo usuário. Assim, estes novos componentes podem ser incorporados às simulações em tempo real, interagindo com os componentes já fornecidos pelo RTDS ${ }^{\mathrm{TM}}$.

\subsection{Implementação em Hardware via DSP}

Neste trabalho, o DSP TMS320F2812 (TEXAS INSTRUMENTS, 2001), apresentado na figura 12, foi utilizado para implementação do dispositivo responsável pelo controle do chaveamento de linhas de transmissão. Sua plataforma é a eZdsp ${ }^{\mathrm{TM}}$ F2812 (SPECTRUM DIGITAL INC., 2003), a qual dispõe de 16 canais de entrada com conversores A/D de 12 bits. O nível de tensão dos sinais fornecidos a estes canais de entrada deve ser de 0 a $3 \mathrm{~V}$, independente da forma de onda dos sinais. Os conversores A/D são responsáveis pela digitalização dos sinais analógicos, oriundos do RTDS ${ }^{\mathrm{TM}}$, relativos aos sinais de tensão necessários ao funcionamento do método proposto.

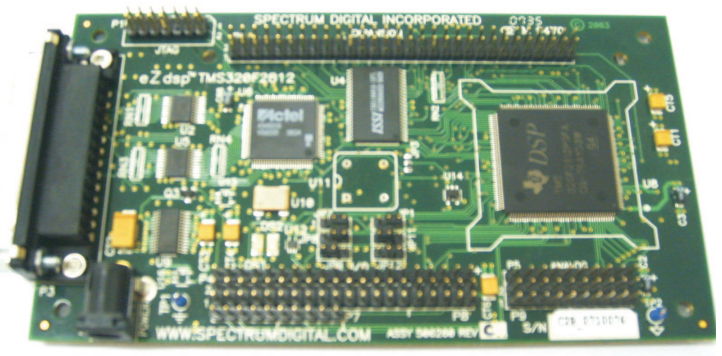

Figura 12: Plataforma eZdsp ${ }^{\mathrm{TM}}$ F2812.

O RTDS ${ }^{\mathrm{TM}}$ apresenta interfaces para entrada e saída de dados do sistema elétrico, permitindo a comunicação com equipamentos externos, bem como a simulação em malha fechada do sistema elétrico de potência juntamente com dispositivos de controle e de proteção (Forsyth et al., 2004). A comunicação entre o RTDS ${ }^{\mathrm{TM}}$ e os dispositivos externos é feita por meio de uma família de cartões denominada GT-I/O (Gigabit Transceiver - Input/Output). Neste trabalho serão utilizados os seguintes cartões:

- Gigabit Transceiver Analogue Output Card (GTAO): Este cartão é usado para gerar sinais analógicos com valores de pico entre $\pm 10 \mathrm{~V}$. Essas saídas são usadas para monitorar os sinais de referência para o chaveamento controlado .

- Gigabit Transceiver Digital Output Card (GTDO): Este 
cartão é usado para gerar sinais digitais de 7 a $24 \mathrm{~V}$. Essas saídas são usadas para monitorar o estado do disjuntor: fechado ou aberto.

- Gigabit Transceiver Digital Input Card (GTDI): Este cartão é usado como interface de entrada para sinais digitais $(0-5 \mathrm{~V})$ provenientes de equipamentos externos ao RTDS $^{\mathrm{TM}}$. Essas entradas são utilizadas pelo dispositivo de controle para comandar o fechamento dos disjuntores.

Para adequar os níveis de tensão dos sinais analógicos oriundos do cartão GTAO do RTDS ${ }^{\mathrm{TM}}$ aos níveis de tensão dos canais de entrada dos conversores A/D do DSP, é necessário o condicionamento desses sinais. Este condicionamento foi feito através de um circuito eletrônico simples, confeccionado em uma placa de circuito impresso (Cardoso, 2009). Após o condicionamento, um filtro analógico passa-baixas de terceira ordem do tipo Butterworth, com frequência de corte de $250 \mathrm{~Hz}$, foi utilizado para minimizar o efeito de aliasing e atenuar componentes de alta frequência. Este valor para a freqüência de corte deve-se a tolerâncias e limitações de valores comerciais dos dispositivos eletrônicos (resistores, capacitores e circuitos integrados - CIs) utilizados no projeto do filtro. Em seguida, os sinais foram amostrados pelos conversores A/D do DSP a uma taxa de $6 \mathrm{kHz}$, e fornecidos ao Sistema de Controle do Chaveamento.

Ainda, o DSP possui saídas em forma de sinais PWM (Pulse Width Modulation), os quais são utilizados para enviar o comando para o fechamento dos disjuntores através do cartão GTDI do RTDS ${ }^{\mathrm{TM}}$. Essas saídas são controladas por registradores e podem ter seu estado alterado em qualquer momento, permitindo assim, o envio do comando para os disjuntores imediatamente após os instantes apropriados para o chaveamento serem calculados.

\section{AVALIAÇÃO DO MÉTODO}

O método ora apresentado foi avaliado através de simulações digitais de energizações e religamentos tripolar de linhas de transmissão em aberto, considerando diferentes condições de operação para o chaveamento e fazendo uso do RTDS ${ }^{\mathrm{TM}}$.

\subsection{Sistema Elétrico Utilizado}

Baseando-se em dados reais obtidos do sistema elétrico de potência de $500 \mathrm{kV}$ da Companhia Hidro Elétrica do São Francisco (CHESF), um sistema elétrico simplificado foi elaborado para avaliação do método (figura 13). Este sistema foi modelado no RTDS ${ }^{\mathrm{TM}}$, fazendo uso da ferramenta Draft do RSCAD, e consiste em uma linha de transmissão de $400 \mathrm{~km}$ de extensão com pára-raios e reatores em derivação de 200
Mvar em cada extremidade, o que corresponde a aproximadamente $77 \%$ de compensação reativa da linha. Ainda, este sistema possui duas fontes com suas respectivas impedâncias conectadas às extremidades da referida linha por meio de disjuntores. Os dados deste sistema são apresentados nas Tabelas 1,2 e 3 . A tensão da Fonte 1 foi ajustada para a máxima tensão operativa definida para um sistema de $500 \mathrm{kV}$, ou seja, $550 \mathrm{kV}$ (ONS, 2002). Este valor foi considerado 1,0 p.u. por ser comumente adotado como base em estudos de sobretensões de manobras no SIN (EPE, 2006).

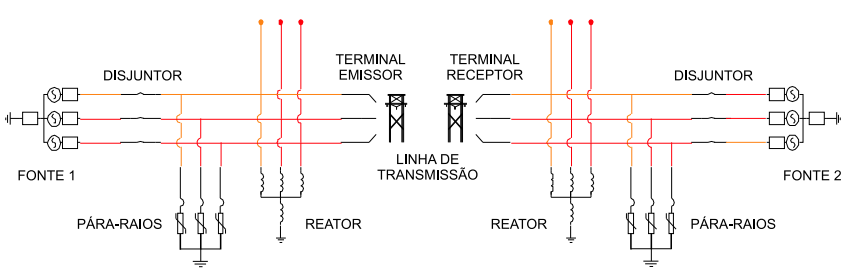

Figura 13: Sistema elétrico modelado no RTDS ${ }^{\mathrm{TM}}$.

Tabela 1: Dados de sequência da linha de transmissão.

\begin{tabular}{cccc}
\hline Sequência & $R(\Omega / \mathrm{km})$ & $X(\Omega / \mathrm{km})$ & $\omega C(\mu \mho / \mathrm{km})$ \\
\hline \hline Zero & 0,3996 & 0,9921 & 3,0839 \\
\hline Positiva & 0,0333 & 0,3170 & 5,2033 \\
\hline
\end{tabular}

Tabela 2: Tensão das fontes $\left(V_{b a s e}=550 \mathrm{kV}\right)$.

\begin{tabular}{ccc}
\hline Barra & Módulo (p.u.) & Fase $\left(^{\circ}\right)$ \\
\hline \hline Fonte 1 & 1,00 & 0 \\
\hline Fonte 2 & 0,99 & -10 \\
\hline
\end{tabular}

Tabela 3: Impedâncias das Fontes.

\begin{tabular}{ccccccc}
\hline \multirow{2}{*}{ Fonte } & \multicolumn{2}{c}{ Sequência zero } & & \multicolumn{2}{c}{ Sequência positiva } \\
\cline { 2 - 3 } \cline { 5 - 6 } & $R_{0}(\Omega)$ & $X_{0}(\Omega)$ & & $R_{1}(\Omega)$ & $X_{1}(\Omega)$ \\
\hline \hline Fonte 1 & 1,1268 & 20,838 & & 0,9681 & 28,513 \\
\hline Fonte 2 & 1,1268 & 20,838 & & 0,9681 & 28,513 \\
\hline
\end{tabular}

Nas simulações foram utilizados pára-raios de óxido de zinco ( $\mathrm{ZnO}$ ) com tensão nominal de $420 \mathrm{kV}$ e um nível de proteção a impulso de manobra de $830 \mathrm{kV}$ em $2 \mathrm{kA}$. Este tipo de pára-raios é comumente especificado para sistemas de 500 kV e são conectados nos terminais das linhas de transmissão (Esmeraldo et al., 1999). 


\subsection{Procedimento de Análise}

Para uma correta avaliação do chaveamento controlado de linhas de transmissão, é necessário que os disjuntores sejam representados de forma adequada, uma vez que estes equipamentos apresentam variações nos respectivos tempos de operação. Uma descrição dos valores típicos para estas variações em diferentes tipos de disjuntores pode ser encontrada em CIGRE Task Force 13.00.1 (1995). Devido às incertezas usuais de fechamento hidráulico mecânico, dispersões estatísticas em relação ao tempo de operação dos disjuntores devem ser consideradas. Desta forma, este tempo de operação é dado pela equação (8):

$$
T_{\text {operacao }}=T_{\text {nominal }}+\Delta T_{\text {estatistico }} .
$$

Onde,

- $T_{\text {nominal }}$ : tempo nominal de operação do disjuntor utilizado na Lógica do Controlador.

- $\Delta T_{\text {estatistico }}$ : variação estatística do tempo de operação do disjuntor.

Dispersões estatísticas no tempo de operação podem ocorrer mesmo em condições idênticas de operação e representam uma limitação inerente ao método de chaveamento controlado. Estas dispersões foram representadas no RTDS ${ }^{\mathrm{TM}}$ por uma distribuição de probabilidade Gaussiana descrita por um desvio padrão $(\sigma)$. Segundo CIGRE Working Group 13.07 (1999a), a máxima dispersão pode ser dada em função do desvio padrão através da equação (9). Assim, considerando uma dispersão máxima no tempo de operação do disjuntor de $2 \mathrm{~ms}$, obtém-se um desvio padrão de aproximadamente 0,67 $\mathrm{ms}$. Este valor de $2 \mathrm{~ms}$ para a dispersão máxima foi adotado por ser um valor conservativo utilizado em estudos de aplicabilidade do chaveamento controlado (CIGRE Working Group 13.07, 1999a).

$$
\Delta T_{\text {estatistico }}=3 \sigma .
$$

Outra característica importante a ser considerada é a taxa de decaimento da suportabilidade dielétrica dos disjuntores $\left(d v_{d i s j} / d t\right)$, a qual pode ser aproximada por uma função linear no tempo, nos instantes em que os contatos do disjuntor estão próximos de se acoplarem fisicamente durante a manobra de fechamento. Neste trabalho, considerou-se $d v_{d i s j} / d t$ maior que o máximo valor da derivada da tensão do sistema, o que evita a ocorrência do pré-arco (Esmeraldo et al., 1999). No entanto, para análise de um disjuntor específico, $d v_{d i s j} / d t$ deve ser modelada apropriadamente.
$\mathrm{Na}$ avaliação do método, diferentes condições de operação para o chaveamento da LT em questão foram avaliadas considerando o terminal a esquerda como o terminal líder (primeiro terminal a ser religado), um tempo morto de $500 \mathrm{~ms}$ para as manobras de religamento tripolar e o sistema íntegro. A seguir são apresentadas as condições abordadas para as manobras de energização e religamento tripolar:

- Chaveamento com reatores em ambos os terminais (aproximadamente 77\% de compensação);

- Chaveamento com reatores apenas no terminal líder (aproximadamente 38\% de compensação).

Além disto, o desempenho do chaveamento controlado (implementado em software e em hardware) é comparado com o dos tradicionais resistores de pré-inserção, onde uma resistência de $400 \Omega$ e um tempo de inserção de $8 \mathrm{~ms}$ foram usados nas simulações por serem valores típicos implementados no SIN. A determinação do valor ótimo para o resistor se dá através de estudos de sobretensões ao longo da LT devido às manobras de inserção e de curto-circuito do mesmo. Nas simulações não foram observadas diferenças significativas no perfil de tensão ao longo da LT ao se utilizar valores de resistores de pré-inserção entre 200 e $400 \Omega$. Ainda, para fins de comparação, também são avaliadas situações em que não há aplicação de nenhum dos dois métodos acima, ou seja, há apenas os pára-raios nas extremidades da linha.

\subsection{Resultados}

Para cada caso avaliado, um total de 100 simulações foram realizadas considerando as dispersões estatísticas no tempo de operação do disjuntor. Destas simulações, os máximos valores de sobretensões ao longo da linha de transmissão, os quais têm probabilidade de ocorrência menor ou igual a $2 \%$, são apresentados na figura 14 para manobras de energização e religamento tripolar. Estes valores estatísticos são normalmente utilizados para a coordenação de isolamento de sistemas elétricos (IEEE Std 1313.1-1996, 1996). As sobretensões são avaliadas nos terminais da linha e a 25, 50 e $75 \%$ do seu comprimento total. Observa-se que a utilização do RPI ou do chaveamento controlado (via CBuilder ou DSP) em conjunto com os pára-raios limitam as sobretensões de manobra de forma eficiente, atingindo níveis de sobretensões bem inferiores àqueles atingidos apenas com pára-raios.

A fim de facilitar a análise comparativa entre os métodos utilizados para limitação de sobretensões de manobra na linha de transmissão em questão, são apresentados na Tabela 4 os máximos valores de sobretensão com probabilidade de ocorrência menor ou igual a $2 \%$ para cada uma das condições de operação abordadas. 


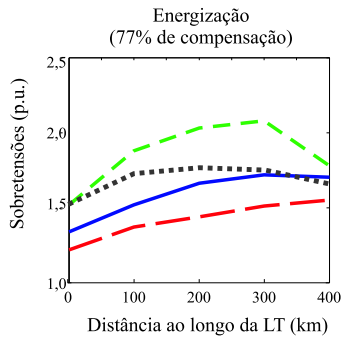

(a)

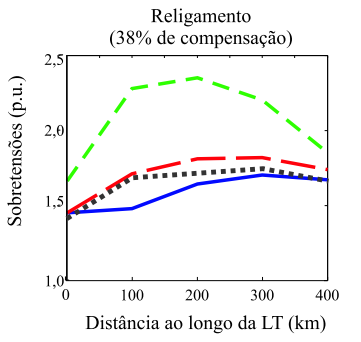

(c)

Figura 14: Sobretensões ao longo da LT: (a) Energização (77\% de compensação); (b) Religamento (77\% de compensação); (c) Religamento (38\% de compensação)

Tabela 4: Máximos valores de sobretensão com probabilidade de ocorrência menor que $2 \%($ Vbase $=550 \mathrm{kV})$.

\begin{tabular}{lcccc}
\hline \multirow{2}{*}{$\begin{array}{c}\text { Condição } \\
\text { de operação }\end{array}$} & PR & RPI-PR & CCS-PR & CCH-PR \\
\cline { 2 - 5 } & \multicolumn{4}{c}{ Máxima sobretensão (p.u.) } \\
\hline $\begin{array}{c}\text { Energização } \\
(77 \% \text { de compensação ) }\end{array}$ & 2,08 & 1,55 & 1,72 & 1,74 \\
\hline $\begin{array}{c}\text { Religamento } \\
\text { de compensação ) }\end{array}$ & 2,45 & 1,78 & 1,67 & 1,68 \\
\hline $\begin{array}{c}\text { Religamento } \\
(38 \% \text { de compensação ) }\end{array}$ & 2,41 & 1,84 & 1,76 \\
\hline $\begin{array}{l}\text { PR - Pára-raios. } \\
\text { RPI-PR - Resistor de pré-inserção e pára-raios. } \\
\text { CCS-PR - Chaveamento controlado (em software) e pára-raios. } \\
\text { CCH-PR - Chaveamento controlado (em hardware) e pára-raios. }\end{array}$
\end{tabular}

Com base nos dados expostos, pode-se observar que na situação mais favorável para o chaveamento controlado, implementado em software, as sobretensões são limitadas a valores não superiores a 1,67 p.u. e na situação mais adversa, as sobretensões são limitadas a 1,72 p.u. Para o caso de implementação do chaveamento controlado em hardware, na situação mais favorável, as sobretensões são limitadas a 1,68 p.u., enquanto que na situação mais adversa, as sobretensões são limitadas a 1,76 p.u. A diferença entre os resultados das implementações em software e hardware é justificada pela imprecisão inerente ao processo de aquisição e tratamento dos sinais analógicos provenientes do RTDS $^{\mathrm{TM}}$.
Com relação ao desempenho do resistor de pré-inserção, na situação mais favorável, as sobretensões são limitadas a 1,55 p.u., enquanto que na situação mais adversa, as sobretensões são limitadas a 1,84 p.u. Assim, de acordo com as simulações digitais em tempo real, o uso do chaveamento controlado pode eliminar a necessidade da utilização de resistores de pré-inserção.

Os resultados obtidos com o método proposto se devem ao processo de estimação dos sinais de referência em instantes futuros, o qual possibilita a determinação de instantes apropriados para o fechamento dos disjuntores. Nas figuras 15 e 16, as quais foram obtidas por meio de simulações digitais, são apresentados respectivamente os sinais de referência, simulados e estimados pelo método, para o religamento da linha com 38\% e 77\% de compensação.

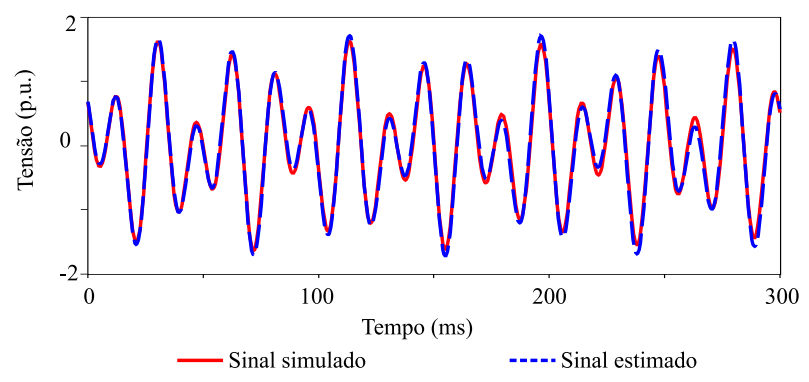

Figura 15: Sinais de tensão entre os contatos do disjuntor para a LT com $38 \%$ de compensação.

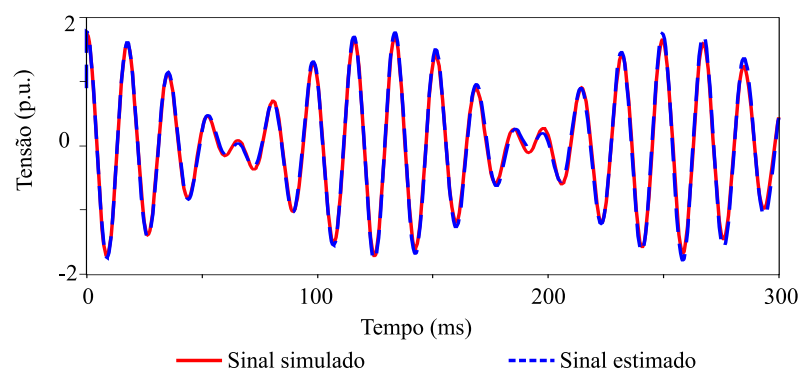

Figura 16: Sinais de tensão entre os contatos do disjuntor para a LT com $77 \%$ de compensação.

A partir destas figuras, observa-se a conformidade entre os sinais simulados e os sinais estimados pelo método proposto, durante um período de $300 \mathrm{~ms}$. Em geral, o instante apropriado para a realização do chaveamento controlado encontra-se dentro deste intervalo, uma vez que o chaveamento normalmente ocorre no primeiro ou segundo período de menor batimento do sinal de referência, tal como indicado nas figuras 4 e 5 . 
O desempenho do método proposto com relação à determinação do instante exato de passagem por zero do sinal de referência apresenta um erro que varia aleatoriamente. Este erro pode ser verificado com o auxílio de sinais tais como os apresentados nas figuras 15 e 16. Baseando-se nestas figuras, observa-se nos sinais simulados os instantes ideais para o chaveamento do disjuntor e a partir dos instantes determinados pelo método estima-se o erro em questão.

Com base neste procedimento, diversas simulações digitais de manobras de religamento da linha de transmissão em questão foram realizadas e o erro médio em relação à determinação dos instantes apropriados para o chaveamento foi de $0,4 \mathrm{~ms}$. Os erros máximos e mínimos foram, respectivamente, cerca de 1,6 ms e $10 \mu$ s. Estes erros são aceitáveis, uma vez que o método busca a passagem por zero em um período de batimento mínimo do sinal de referência e portanto não ocasiona sobretensões elevadas. Já com relação às manobras de energização, erros insignificantes da ordem de 10 $\mu$ s são verificados.

\section{CONCLUSÕES}

Um método para chaveamento controlado de linhas de transmissão foi apresentado e avaliado, pela primeira vez, por meio de simulações digitais em tempo real via RTDS ${ }^{\mathrm{TM}}$. Além disso, foi desenvolvido um protótipo de um dispositivo capaz de controlar o fechamento dos contatos dos disjuntores. A possibilidade de implementação do método em tempo real, tanto em software quanto em hardware, devese ao fato de que na estratégia de chaveamento apresentada, foram utilizadas apenas técnicas simples de tratamento de sinais a exemplo de detecção de passagens por zero.

Os resultados obtidos comprovam a eficiência do método em limitar as sobretensões de manobra (energização e religamento tripolar) em LTs, com desempenho similar nas duas formas de implementação abordadas: em software (via CBuilder) e em hardware (via DSP). De forma geral, na situação mais favorável ao chaveamento controlado, as sobretensões foram limitadas a 1,67 p.u., enquanto que na situação mais adversa, as sobretensões foram limitadas a 1,76 p.u.

Com relação ao desempenho do resistor de pré-inserção, na situação mais favorável, as sobretensões foram limitadas 1,55 p.u., enquanto na situação mais adversa, estas foram limitadas a 1,84 p.u. Com isto, conclui-se que o chaveamento controlado pode eliminar a necessidade do uso dos resistores de pré-inserção, aumentando assim a confiabilidade dos disjuntores de linhas e reduzindo os seus custos de fabricação e manutenção. Ainda, o chaveamento controlado pode reduzir os transitórios originados a partir de manobras no sistema elétrico, propiciando uma melhoria na qualidade da energia e um consequente aumento na vida útil dos equipamentos.
A utilização do chaveamento controlado em conjunto com pára-raios localizados nas extremidades das linhas de transmissão propicia ainda segurança em caso de falha do Sistema de Controle do Chaveamento. Porém, uma vez que diversos parâmetros do sistema elétrico podem influenciar nos níveis de sobretensões de manobras, devido às particularidades de cada sistema e das respectivas linhas de transmissão, os resultados ora obtidos, a priori não devem ser generalizados e uma análise detalhada da aplicação do método apresentado deve ser realizada para cada sistema. Dentre os parâmetros que devem ser levados em consideração nas analises encontram-se a geometria da linha de transmissão e o respectivo esquema de transposição, além das especificações dos disjuntores com relação à variação no tempo de operação de fechamento e taxa de decaimento da rigidez dielétrica do mesmo.

\section{AGRADECIMENTOS}

Os autores agradecem ao CNPq pelo apoio financeiro para a realização desta pesquisa e aos revisores pelas valiosas sugestões.

\section{REFERÊNCIAS}

ABB (2009). Controlled Switching, Buyer's \& Application Guide, 3 edn, ABB, Ludvika, Sweden.

Cardoso, G. A. (2009). Chave Controlada para Redução de Sobretensões de Manobra em Linhas de Transmissão, Master's thesis, Universidade Federal de Campina Grande, Campina Grande, Brasil.

CIGRE Task Force 13.00.1 (1995). Controlled Switching: A State of the Art Survey - Part I, ELECTRA (162): 6597.

CIGRE Task Force 13.00.1 (1996). Controlled Switching: A State of the Art Survey - Part II, ELECTRA (164): 3961.

CIGRE Working Group 13.07 (1999a). Controlled Switching of HVAC Circuit Breakers: Guide for Application Lines, Reactors, Capacitors, Transformers - 1st Part, ELECTRA (183): 42-73.

CIGRE Working Group 13.07 (1999b). Controlled Switching of HVAC Circuit Breakers: Guide for Application Lines, Reactors, Capacitors, Transformers - 2nd Part, ELECTRA (185): 36-57.

Dantas, K. M. C., Fernandes Jr., D., Neves, W. L. A., Souza, B. A. and Fonseca, L. C. A. (2008). Mitigation of Switching Overvoltages in Transmission Lines via Controlled Switching, IEEE Power \& Energy Society General Meeting . 
Dantas, K. M. C., Neves, W. L. A., Fernandes Jr., D., Cardoso, G. A. and Fonseca, L. C. A. (2009). On Applying Controlled Switching to Transmission Lines: Case Studies, International Conference on Power Systems Transients (IPST).

Doi, H., Goto, M., Kawai, T., Yokokawa, S. and Suzuki, T. (1990). Advanced Power System Analogue Simulator, IEEE Transactions on Power Systems 5(3): 962-968.

Dommel, H. W. (1996). Electromagnetic Transients Program Reference Manual: EMTP Theory Book, Portland, BPA.

EPE (2006). Estudo de Expansão da Interligação N-NE LT 500 kV Colinas - Ribeiro Gonçalves - São João do Piauí - Milagres. No EPE/GET - NE-R1-004.2006.

Esmeraldo, P. C. V., Filho, J. A., Carvalho, F. M. S., Carva1ho, A. C. C. and Morais, S. A. (1999). Circuit-Breaker Requirements for Alternative Configurations of a 500 $\mathrm{kV}$ Transmission System, IEEE Transactions on Power Delivery 14(1): 169-175.

Forsyth, P., Maguire, T. and Kuffel, R. (2004). Real Time Digital Simulation for Control and Protection System Testing, IEEE 35th Annual Power Electronics Specialists Conference, 2004. PESC 04. 1: 329-335 Vol.1.

Froehlich, K., Hoelzl, C., Stanek, M., Carvalho, A. C., Hofbauer, W., Hoegg, P., Avent, B. L., Peelo, D. F. and Sawada, J. H. (1997a). Controlled Closing on Shunt Reactor Compensated Transmission Lines. Part I: Closing Control Device Development, IEEE Transaction on Power Delivery 12(2): 734-740.

Froehlich, K., Hoelzl, C., Stanek, M., Carvalho, A. C., Hofbauer, W., Hoegg, P., Avent, B. L., Peelo, D. F. and Sawada, J. H. (1997b). Controlled Closing on Shunt Reactor Compensated Transmission Lines. Part II: Application of Closing Control Device for HighSpeed Autoreclosing on BC Hydro 500 kV Transmission Line, IEEE Transaction on Power Delivery 12(2): 741-746.

IEEE Std 1313.1-1996 (1996). IEEE Standard for Insulation Coordination - Definitions, Principles and Rules, IEEE, New York, USA.

Ito, H. (2002). Controlled Switching Technologies, State-ofthe-Art, Transmission and Distribution Conference and Exhibition 2002: Asia Pacific, IEEE/PES .

Kuffel, R., Giesbrecht, J., Maguire, T., Wierckx, R. and McLaren, P. (1995). RTDS - A Fully Digital Power System Simulator Operating in Real Time, First International Conference on Digital Power System Simulators, ICDS '95., 1995.
Mestas, P. and Tavares, M. C. (2007). Comparative Analysis of Control Switching Transient Techniques in Transmission Lines Energization Maneuver, International Conference on Power Systems Transients (IPST) .

ONS (2002). Procedimentos de Rede - Submódulo 23.3. Diretrizes e Critérios para Estudos Elétricos.

Oppenheim, A. V. and Schafer, R. W. (1989). Discrete-Time Signal Processing, Prentice-Hall, New Jersey, USA.

Ouellette, D. S., Wierckx, R. P. and McLaren, P. G. (2008). Using a Multi-Threaded Time-Step to Model a MultiFunction Relay in a Real Time Digital Simulator, IET 9th International Conference on Developments in Power System Protection (DPSP) pp. 162-167.

Schweitzer, E. O. and Hou, D. (1993). Filtering for Protective Relays, Schweitzer Engineering Laboratories, Inc., Pullman, Washington USA.

SPECTRUM DIGITAL INC. (2003). eZdsp F2812 Technical Reference, Stafford, TX - USA.

TEXAS INSTRUMENTS (2001). TMS320F2812 Digital Signal Processor Data Manual, Dallas, TX - USA. Revised July 2007.

Tsutada, H., Hirai, T., Kohyarna, H., Ito, H. and Sasaki, K. (2002). Development of Synchronous Switching Controller for Gas Circuit Breakers, Transmission and Distribution Conference and Exhibition 2002: Asia Pacific. IEEE/PES.

Wagner, C. and Bankoske, J. (1967). Evaluation of Surge Suppression Resistors in High-Voltage Circuit Breakers, Power Apparatus and Systems, IEEE Transactions on PAS-86(6): 698-707. 